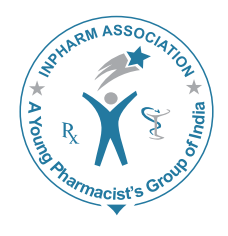

\title{
JVP
}

\section{Preparation and Characterization of Metformin Hydrochloride - Compritol 888 ATO Solid Dispersion}

\author{
Jagdale SC, Patil SA, Kuchekar BS, Chabukswar AR \\ Department of Pharmaceutics, MAEER's Maharashtra Institute of Pharmacy, Pune, Maharashtra, India \\ Address for correspondence: Dr. S. C. Jagdale; E-mail: jagdaleswati@rediffmail.com
}

\begin{abstract}
Metformin hydrochloride (MET) sustained-release solid dispersions (SD) were prepared by the solvent evaporation and closed melt method, using compritol 888 ATO as the polymer with five different drug-carrier ratios. Characterization of solid dispersion was carried out by Fourier Transform Infrared (FTIR) spectroscopy, ultraviolet (UV) spectroscopy, Differential scanning calorimetry (DSC), X-ray powder diffraction (XRPD). The FTIR and UV studies suggested that no bond formation had occurred between the polymer and the drug. DSC and XPRD results ruled out any interaction or complex formation between the drug and the polymer. The formulated SD had acceptable physicochemical characters and SD with a 1:4 drug : Polymer ratio, which released the drug over an extended period of eight-to-ten hours. The data obtained from the in vitro release studies were fitted with various kinetic models and were found to follow the Korsmeyer-Peppas equation. The prepared SD showed good stability over the studied time period. The solvent evaporation method was found to be more helpful than the closed melt method, giving the sustained release action. The SD with a $1: 4$ ratio of drug to polymer, by the solvent evaporation method, was selected as the most effective candidate for the subsequent development of a well-timed, sustained-release dosage form of the drug.
\end{abstract}

Key words: Closed melt, compritol 888 ATO, metformin, solid dispersion, solvent evaporation

\section{INTRODUCTION}

Metformin hydrochloride (MET) is a highly water-soluble anti-hyperglycemic agent used in the treatment of type 2 non-insulin-dependent diabetes mellitus. Its relatively low $(50-60 \%)$ bioavailability, together with its short and variable biological half-life $(1.5-4.5$ hours $),{ }^{[1]}$ require

\begin{tabular}{|l|l|}
\hline \multicolumn{2}{|c|}{ Access this article online } \\
\hline Quick Response Code: & \multirow{2}{*}{ Website: } \\
\hline & www.jyoungpharm.in \\
& DOI: \\
\hline
\end{tabular}

repeated administrations of high doses to maintain effective plasma concentrations, thus reducing patient compliance and/or enhancing the incidence of side-effects. Side effects and the need for administration, twice or thrice a day when larger doses are required, can decrease patient compliance. Sustained release (SR) formulation that would maintain the plasma levels of the drug for eight to twelve hours might be sufficient for once daily dosing of metformin. Administration of a sustained-release, oncea-day metformin dosage form could reduce the dosing frequency and improve patient compliance..$^{[2]}$ Sustained or controlled drug delivery occurs when embedded within a polymer that may be natural or semi-synthetic or synthetic in nature. The polymer is judiciously combined with the drug or other active ingredients in such a way that the active 
agent is released from the material in a predetermined fashion and releases the drug at a constant rate for the desired time period. ${ }^{[3]}$

Solid dispersion (SD), in which compounds are dispersed into water-soluble carriers, has been generally used to improve the dissolution properties and the bioavailability of drugs that are poorly soluble in water. ${ }^{[4]}$ Solid dispersion has also been applied for the controlled release of drugs. Previous reports have shown that by using solid dispersions it is possible to precisely control the rate of release of an extremely water soluble drug, such as oxprenolol hydrochloride ${ }^{[5]}$ and that of phenacetin ${ }^{[6]}$ and diclofenac sodium $^{[7]}$ as well. These studies have shown that there is a linear relationship between the rate of release.

Polymers that primarily form insoluble or skeleton matrices are considered as the first category of retarding materials. The second class represents hydrophobic and water insoluble materials, which are potentially erodible and the third group exhibits hydrophilic properties. ${ }^{[8]}$ The drug release for extended duration, particularly for highly watersoluble drugs, using a hydrophilic matrix system is restricted due to the rapid diffusion of the dissolved drug through the hydrophilic gel network. For such drugs with high water solubility, hydrophobic polymers (waxes) are suitable as matrix forming agents for developing sustained-release dosage forms. Hydrophobic polymers provide several advantages, ranging from good stability at varying $\mathrm{pH}$ values and moisture levels, to well-established safe applications. ${ }^{[9]}$

Over the past decade, glyceryl behenate has been used for controlled-release applications by direct compression ${ }^{[10]}$ and more recently by : Hot-melt coating, ${ }^{[1]]}$ melt granulation ${ }^{[12]}$ or pelletization. ${ }^{[13]}$ This glyceride mixture is known to exhibit a complex polymorphism depending on many parameters such as crystallization rate or temperature of storage. ${ }^{[12]}$ Compritol 888 ATO consists of a mixture of mono-, di- and tribehenates of glycerol (18, 52, and 28\% in weight, respectively) and presents a drop point ranging from $69^{\circ} \mathrm{C}$ to $74^{\circ} \mathrm{C}$ and a hydrophilic-lipophilic balance value of 2. More recently, this mixture of glycerides has been designed to provide sustained release of drugs. Such release would not be obtained from more defined compounds like pure di - or triglycerides. ${ }^{[14]}$

Therefore, in the present study, solid dispersions were prepared using compritol 888 ATO by different methods, that is, physical mixing, closed melt method, and the solvent evaporation method, and characterized by differential scanning calorimetry (DSC), X-ray powder diffractometry (PXRD), and Fourier transform infrared spectroscopy
(FTIR) in order to carefully investigate and compare the physical-chemical properties of the obtained dispersions, for a rational selection of the best one. In addition, solid dispersions were examined for a drug release pattern and release mechanism.

\section{MATERIALS AND METHODS}

\section{Materials}

Metformin hydrochloride was kindly supplied by Indoco Remedies (Goa, India) and glyceryl behenate (Compritol 888 ATO) were obtained from Colorcon Asia Pvt. Ltd (Mumbai, India). All other chemicals and solvents were of reagent grade.

\section{Preparation methods}

\section{Preparation ol physical mixtures}

Physical mixtures (PM) ${ }^{[15]}$ of MET and compritol in powder form were mixed in mortar and passed through sieve mesh No. 60. The PM were prepared in the following ratios : MET : Compritol in the ratios of $1: 1,1: 2,1: 3$, $1: 4$, and $1: 5$.

\section{Solis dispersions prepared by the solvent evaporation method ${ }^{[16]}$}

Solid dispersions were prepared by dissolving accurately weighed amounts of compritol and MET in chloroform. After complete dissolution, the solvent was left to evaporate in open air for two days. Subsequently, the solid mass was ground and passed through sieve No. 60. The sieved ground powders were stored at $25^{\circ} \mathrm{C}$ in a desiccator, in a screw-capped glass vial until use.

\section{Solis dispersions prepared by the closed melt method}

Solid dispersions were prepared by the closed melting method $^{[17]}$ as follows. From the PMs, $2 \mathrm{~g}$ were placed into each ampule and then sealed. The ampules were then heated at $80^{\circ} \mathrm{C}$ for 10 minutes. Subsequently they were opened and dried for 10 minutes at each heating temperature, in order to remove the water. The samples were collected from the ampules and kept overnight, triturated, and passed through a 60 No. sieve. The solid dispersions were then stored in air tight containers until evaluation.

Evaluation and characterization of solid dispersion

\section{Drut content and percent yield}

Physical mixtures and solid dispersions equivalent to $10 \mathrm{mg}$ of MET were weighed accurately and dissolved in $25 \mathrm{ml}$ of methanol. The solutions were filtered through a membrane filter $(0.45 \mathrm{~mm})$. The drug content was determined at 
$232 \mathrm{~nm}$ by UV spectrophotometer (Varian Cary 100) after suitable dilution in distilled water (DW). The percentage yield of each formulation was also calculated. ${ }^{[18]}$

\section{Spectroscopic studies}

Drug polymer interactions between MET and compritol was studied by the spectral shift method. Ten milligrams of drug and solid dispersions equivalent to $10 \mathrm{mg}$ MET were dissolved in $25 \mathrm{ml}$ methanol, filtered using Whatman filter paper No.41 and degassed by sonication for 30 minutes. After appropriate dilutions using DW, the solutions were scanned at $232 \mathrm{~nm}$ with a UV spectrophotometer (Varian Cary 100). ${ }^{[1]}$

\section{Dissolution study}

The dissolution studies ${ }^{[20]}$ were performed using a US Pharmacopoeia 24 type II dissolution test apparatus (Electrolab TDT-08L, Mumbai, India). The samples equivalent to $10 \mathrm{mg}$ MET were placed in a dissolution vessel containing $900 \mathrm{ml}$ of double distilled water maintained at $37 \pm 0.5^{\circ} \mathrm{C}$ and stirred at $100 \mathrm{rpm}$. Fivce milliliter samples were collected periodically and replaced with a fresh dissolution medium. After filtration through Whatman filter paper No.41, a concentration of MET was determined spectrophotometrically at $232 \mathrm{~nm}$. The data were analyzed by PCP Disso software (version 3.0). Solid dispersions were optimized based on the percentage drug release criteria fixed by USP XXXII ${ }^{[21]}$ as given below:

Release at one hour :25 to $40 \%$

Release at two hours :35 to $55 \%$

Release at six hours :65 to $85 \%$

Release at ten hours :Not less than 85\%

\section{Dissolution efficiency}

The dissolution efficiency (DE) ${ }^{[22]}$ of various solid dispersions was calculated. DE is used as the criterion for comparing the effect of polymer concentration on the release rate. Dissolution efficiency (equation 1) is defined as the area under the dissolution curve up to the time, $t$, expressed as a percentage of the area of the rectangle described by $100 \%$ dissolution in the same time.

$$
\mathrm{DE}=\frac{\int_{0}^{\mathrm{T}} \mathrm{Y} \times \mathrm{dt}}{\mathrm{Y}_{100} \times \mathrm{T}} \times 100 \%
$$

where $\mathrm{Y}$ is the percent drug release as the function of time, $\mathrm{t}$. $\mathrm{T}$ is the total time of drug release and $\mathrm{Y}_{100}$ is $100 \%$ drug release.

\section{Release experiments}

In order to gain insight into the drug release mechanism from the solid dispersion, release data ${ }^{[23]}$ of selected formulations were examined according to the zero-order, first-order, and the Higuchi's square root of time mathematical models, Hixson and Crowell powder dissolution method, Korsmeyer and Peppas model, and the release exponent, n, was calculated. All the dissolution profiles were subjected to model fitting using PCP Disso software (version 3.0).

An $\mathrm{n}$ value 0.5 is considered consistent with a diffusioncontrolled release, whereas, a value of 1.0 indicates a zero-order release behavior, and intermediate values $(0.5>n>1.0)$ are defined as anomalous non-fickian transport mechanism. ${ }^{[2]}$

Similarity factor (f2) analysis

In vitro release profile of the marketed metformin-sustained release tablets, (Glumet XR ${ }^{\circledR}$, Cipla) was performed under similar conditions as used for in vitro release testing of the test product for the release of MET. The similarity factor ${ }^{[25]}$ between the two formulations was determined using the data obtained from the drug release studies. The data was analyzed by the formula shown in equation 2 .

$\mathrm{f} 2=50 \log \left\{\left[1+(1 / \mathrm{N}) \sum(\mathrm{Ri}-\mathrm{Ti})^{2}\right]^{-0.5} \times 100\right\}$

where $\mathrm{N}=$ number of time points, $\mathrm{Ri}$ and $\mathrm{Ti}=$ dissolution of reference and test products at time i. If $\mathrm{f} 2$ is greater than 50 it is considered that the two products share similar drug release behaviors. ${ }^{[26]}$

\section{Diffuse reflectance infrared Fourier transform spectroscopy}

The diffuse reflectance infrared Fourier transform spectroscopy (DRIFTS) ${ }^{[27]}$ spectra of pure metformin, physical mixtures, and solid dispersions were obtained, after appropriate background subtraction, using an FTIR spectrometer (FTIR-640 IR, Varian). About 2 to $3 \mathrm{mg}$ of the sample was mixed with dry potassium bromide, and the sample was scanned from 4,000 to $400 \mathrm{~cm}^{-1}$.

\section{Differential scanning calorimetry}

The calorimeter used was DSC 823e (Mettler Toledo, Switzerland), which was equipped with an intracooler and a refrigerated cooling system. The Indium standard was used to calibrate the DSC temperature and enthalpy scale. Nitrogen was used to purge gas through the DSC cell at the flow rate of $50 \mathrm{ml} /$ minute and $100 \mathrm{ml} /$ minute through the cooling unit. The samples $(5-10 \mathrm{mg}$ were hermetically sealed in an aluminum pan and the heating rate was carried out at $5^{\circ} \mathrm{C} /$ minute. ${ }^{[28]}$

\section{$X$-ray powder diffractometry}

The powder X-ray diffraction patterns ${ }^{[2]}$ of the powdered samples were recorded by using a Philips PW-1729 $\mathrm{X}$-ray diffractometer. Samples were irradiated with monochromatized $\mathrm{CuK} \alpha$ radiation and a graphite 
monochromator. The samples were analyzed in the $5-50^{\circ}$ $2 \theta$ range at a scan rate of $0.05^{\circ}$ per second.

\section{Stability studies}

The stability studies ${ }^{[30]}$ were conducted on metformin solid dispersions along with the physical mixtures, to assess their stability with respect to their physical appearance, drug content, and drug release characteristics after storing them at $40^{\circ} \mathrm{C}$ and relative humidity (RH) of $75 \%$ for one month and at room temperature, for six months.

\section{RESULTS}

\section{Preparation of solid dispersions}

Table 1 summarizes the abbreviations, drug content, and yield along with dissolution efficiency for the solid dispersions. The production yields of solid dispersions from solvent evaporation ranged between $91.87 \pm 0.55 \%$ and $97.80 \pm 1.99 \%$.

The production yields of solid dispersions from the closed melt method ranged between $98.15 \pm 1.19 \%$ and $99.20 \pm 0.17 \%$. The amount of drug determined in each solid dispersion was between $96.70 \pm 0.31 \%$ and $99.56 \pm 1.10 \%$ for the solvent evaporation method and $96.05 \pm 0.64 \%$ and $98.56 \pm 0.57 \%$ for the closed melt method.

\section{Spectroscopic studies}

The UV spectra of MET and solid dispersions were studied. There was no shift in the $\lambda$ max of metformin in the presence of compritol.

\section{Dissolution study}

The dissolution profiles of metformin solid dispersions, along with physical mixtures, in double distilled water, are shown in Figures 1 and 2, for solvent evaporation and the closed melt method, respectively. MET is a highly aqueous soluble and got completely dissolved within a few minutes. The dissolution from the physical mixture showed

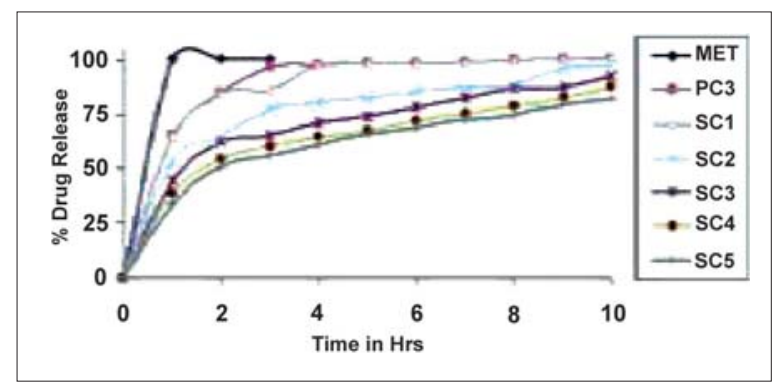

Figure 1: Dissolution profile of metformin hydrochloride, physical mixture, and solid dispersions using the solvent evaporation method approximately the same behavior of pure MET, with only a very slight initial slowing down of the drug dissolution rate, due to the presence of the hydrophobic polymer, which reduced the drug wettability. As the ratio of drug to polymer increased there was a significant decrease in the drug release from the solid dispersions, reaching $80 \%$ of dissolved drug after about four and seven hours for SC2 and SC3. As the quantity of polymer increased further there was

Table 1: Abbreviations, drug content, yield, and dissolution efficiency for the solid dispersions

\begin{tabular}{|c|c|c|c|c|}
\hline $\begin{array}{l}\text { Method of } \\
\text { preparation }\end{array}$ & Abbreviations & $\begin{array}{l}\text { \% drug } \\
\text { content }^{a}\end{array}$ & $\%$ yield $^{a}$ & $\begin{array}{c}\text { Dissolution } \\
\text { efficiency }\end{array}$ \\
\hline $\begin{array}{l}\text { Physical } \\
\text { mixture } 1: 1\end{array}$ & PC1 & $98.90 \pm 0.63$ & $99.15 \pm 0.35$ & - \\
\hline $\begin{array}{l}\text { Physical } \\
\text { mixture } 1: 2\end{array}$ & $\mathrm{PC} 2$ & $97.63 \pm 0.51$ & $99.23 \pm 0.24$ & - \\
\hline $\begin{array}{l}\text { Physical } \\
\text { mixture } 1: 3\end{array}$ & $\mathrm{PC} 3$ & $96.72 \pm 0.91$ & $99.35 \pm 0.34$ & - \\
\hline $\begin{array}{l}\text { Physical } \\
\text { mixture } 1: 4\end{array}$ & $\mathrm{PC} 4$ & $97.80 \pm 1.03$ & $97.83 \pm 0.88$ & - \\
\hline $\begin{array}{l}\text { Physical } \\
\text { mixture } 1: 5\end{array}$ & PC5 & $98.56 \pm 1.08$ & $98.17 \pm 1.01$ & - \\
\hline $\begin{array}{l}\text { Solvent } \\
\text { evaporation } \\
1: 1\end{array}$ & SC1 & $99.56 \pm 1.10$ & $97.80 \pm 1.99$ & 88.11 \\
\hline $\begin{array}{l}\text { Solvent } \\
\text { evaporation } \\
1: 2\end{array}$ & $\mathrm{SC} 2$ & $99.0 \pm 0.44$ & $96.92 \pm 1.48$ & 76.67 \\
\hline $\begin{array}{l}\text { Solvent } \\
\text { evaporation } \\
1: 3\end{array}$ & SC3 & $97.0 \pm 0.96$ & $97.67 \pm 0.94$ & 70.09 \\
\hline $\begin{array}{l}\text { Solvent } \\
\text { evaporation } \\
1: 4\end{array}$ & $\mathrm{SC} 4$ & $98.90 \pm 1.33$ & $91.87 \pm 0.55$ & 63.90 \\
\hline $\begin{array}{l}\text { Solvent } \\
\text { evaporation } \\
1: 5\end{array}$ & SC5 & $96.70 \pm 0.31$ & $92.67 \pm 0.81$ & 60.60 \\
\hline $\begin{array}{l}\text { Closed melt } \\
1: 1\end{array}$ & $\mathrm{CC} 1$ & $98.3 \pm 1.11$ & $98.20 \pm 0.29$ & 91.13 \\
\hline $\begin{array}{l}\text { Closed melt } \\
1: 2\end{array}$ & $\mathrm{CC} 2$ & $98.56 \pm 0.57$ & $98.40 \pm 1.77$ & 89.68 \\
\hline $\begin{array}{l}\text { Closed melt } \\
1: 3\end{array}$ & $\mathrm{CC} 3$ & $96.40 \pm 0.66$ & $99.20 \pm 0.17$ & 86.48 \\
\hline $\begin{array}{l}\text { Closed melt } \\
1: 4\end{array}$ & $\mathrm{CC} 4$ & $96.05 \pm 0.64$ & $99.10 \pm 0.19$ & 87.17 \\
\hline $\begin{array}{l}\text { Closed melt } \\
1: 5\end{array}$ & CC5 & $96.52 \pm 0.85$ & $98.15 \pm 1.19$ & 86.28 \\
\hline
\end{tabular}

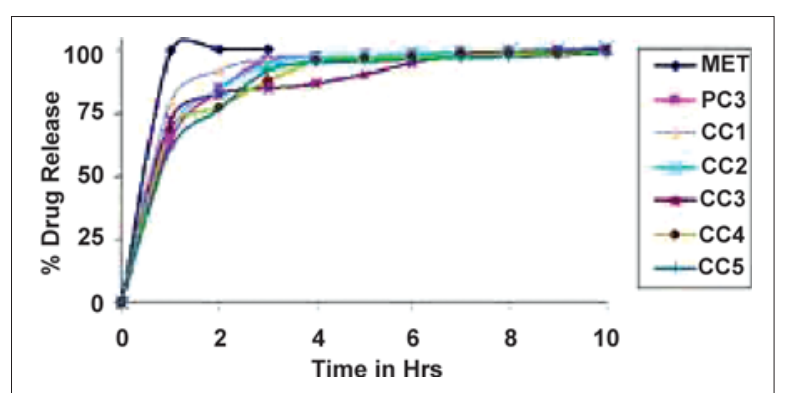

Figure 2: Dissolution profile of metformin hydrochloride, physical mixture, and solid dispersions using the closed melt method 
also a decrease in the amount of drug release from the SD, giving a extended release up to 10 hours for SC4 and SC5.

From the dissolution profile of the solid dispersions of MET with compritol by the closed melt method, as depicted in Figure 2, it is clear that the physical mixture shows approximately the same behavior of pure MET dissolving completely within a few minutes.

\section{Dissolution efficiency}

All the dissolution profiles were subjected to model fitting using PCP Disso software (version 3.0). The dissolution efficiency (DE) of various solid dispersions was calculated [Table 1].

Dissolution efficiency is used as the criterion for comparing the effect of polymer concentration on the release rate. The solid dispersions showing the desired release profile were selected as optimized batches for further evaluations. The solid dispersion of MET with Compritol 888 ATO, which was selected as the optimized batch for further evaluation was SC4 solid dispersion, depending on the release profiles.

\section{Release experiments}

Table 2 shows the data for kinetics of solid dispersions. The in vitro release pattern of the SC4 SDs was analyzed by fitting the dissolution data into various kinetic models. It was observed that the $\mathrm{R}^{2}$ value was higher when fitted to the Korsmeyer-Peppas equation, as compared to the zero order equation, which indicated Peppas as the best fitting kinetic model for SC4.

When the data was plotted according to the first order equation, the SC4 solid dispersions showed a fair linearity, with regression values of 0.9425 . When the data was plotted according to a Hixson and Crowell's equation, the SC4 solid dispersions did not show much linearity, with a regression value of 0.8722 . The solid dispersions did not fit into the zero order equation, with an $\mathrm{R}^{2}$ value of 0.5832 , indicating that the dissolution rate of the drug was not independent of the amount of drug available for dissolution and diffusion from the solid dispersions.

\section{Similarity factor (f2)}

The similarity factor $f 2$ method can be used to compare two dissolution profiles. The reference drug was used. The results of $\mathrm{f} 2$ are shown in Table 3.

Similarity factor analysis between the prepared solid dispersion and marketed tablet for the release of MET showed an $\mathrm{f} 2$ factor greater than $50 ; \mathrm{f} 2=59.55$ for SC4 solid dispersion. The higher the $\mathrm{f} 2$ values, the more similar the dissolution profiles, so $\mathrm{f} 2<50$ represented non-similar profiles, while $\mathrm{f} 2>50$ denoted a similarity between profiles of prepared solid dispersions and the marketed product.

\section{Diffuse reflectance infrared fourier transform spectroscopy}

Two typical bands at $3369 \mathrm{~cm}^{-1}$ and $3294 \mathrm{~cm}^{-1}$, relative to the $\mathrm{N}-\mathrm{H}$ primary stretching vibration, and a band at $3155 \mathrm{~cm}^{-1}$ due to the $\mathrm{N}-\mathrm{H}$ secondary stretching, and characteristic bands at $1626 \mathrm{~cm}^{-1}$ and $1567 \mathrm{~cm}^{-1}$ assigned to C-N stretching are observed in the FTIR spectrum of pure MET [Figure 3a]. However, the IR spectra of compritol show typical bands at $2820 \mathrm{~cm}^{-1}$, due to $\mathrm{C}-\mathrm{H}$ stretching, and $1705 \mathrm{~cm}^{-1}$ due to $\mathrm{C}=\mathrm{O}$ (carbonyl) stretching [Figure $3 \mathrm{~g}$ ]. The physical mixture spectrum [Figure $3 \mathrm{f}$ ] shows all the above bands and can be considered as the sum of drug and carrier. The FTIR spectra of the solid dispersions presented appreciable shifts and reduction in the intensity of the characteristic metformin bands at $3369 \mathrm{~cm}^{-1}, 3294 \mathrm{~cm}^{-1}$, and also at $1626 \mathrm{~cm}^{-1}$, maybe due to the weak hydrogen bonding between the drug and polymer.

\begin{tabular}{|c|c|c|c|}
\hline \multirow[t]{2}{*}{ Model } & \multirow[t]{2}{*}{ Equation } & \multicolumn{2}{|c|}{$\mathrm{SC} 4$} \\
\hline & & $\mathbf{R}^{2}$ & $\mathbf{k}$ \\
\hline Zero order & $\begin{array}{l}\mathrm{F}=\mathrm{k} \times \mathrm{t} \text { (where } \mathrm{F} \\
\text { is the fraction of } \\
\text { drug release, } \mathrm{k} \text { is } \\
\text { the release constant, } \\
\text { and } \mathrm{t} \text { is the time) }\end{array}$ & 0.5832 & 10.7462 \\
\hline First order & $\begin{array}{l}\mathrm{Ln} \mathrm{F}=\mathrm{k} \times \mathrm{t} \text { (where } \\
\mathrm{F} \text { is the fraction of } \\
\text { drug release, } \mathrm{k} \text { is } \\
\text { the release constant, } \\
\text { and } \mathrm{t} \text { is the time) }\end{array}$ & 0.9425 & -0.2112 \\
\hline Higuchi matrix & $\mathrm{F}=\mathrm{k} \sqrt{ } \mathrm{t}$ & 0.9607 & 29.5498 \\
\hline $\begin{array}{l}\text { Hixson and Crowell powder } \\
\text { dissolution method }\end{array}$ & $\begin{array}{l}F=100 \\
\left(1-(1-k t)^{3}\right)\end{array}$ & 0.8722 & -0.0548 \\
\hline Korsmeyer and Peppas model ${ }^{a}$ & $\mathrm{~F}=\mathrm{kt}^{n}$ & 0.9902 & 41.0961 \\
\hline
\end{tabular}

\section{Table 3: f2 factor results}

\begin{tabular}{lccc}
\hline Time & \multicolumn{2}{c}{ Average \% release } & f2 \\
\cline { 2 - 3 } & Reference & SC4 & \\
\hline 0 & 0.00 & 0.00 & 0.00 \\
1 & 28.30 & 39.03 & 55.82 \\
2 & 41.68 & 54.93 & 50.23 \\
3 & 51.92 & 60.33 & 50.99 \\
4 & 59.17 & 64.42 & 52.59 \\
5 & 64.78 & 67.41 & 54.36 \\
6 & 67.34 & 71.71 & 55.50 \\
7 & 71.17 & 75.21 & 56.51 \\
8 & 75.90 & 79.24 & 57.49 \\
9 & 81.35 & 82.87 & 58.56 \\
10 & 86.81 & 87.62 & 59.55 \\
Average & - & - & 59.55 \\
\hline
\end{tabular}




\section{Differential scanning calorimetry}

The thermal curves of MET and of selected solid dispersion SC4, along with the physical mixture, are shown in Figure 4.

The thermal curve of pure MET [Figure 4a] exhibited an initial flat profile followed by a sharp endothermic effect, with a $\mathrm{T}_{\text {peak }}$ at $231.0^{\circ} \mathrm{C}$ and an associated fusion enthalpy of $292.2 \mathrm{~J} / \mathrm{g}$, indicating its anhydrous crystalline state. The DSC profile of Compritol [Figure 4b] showed a sharp endothermic effect, with a $\mathrm{T}_{\text {peak }}$ at $70^{\circ} \mathrm{C}$, due to its crystalline nature. The thermal curve of the physical mixture [Figure 4c] showed a sharp endothermic peak at $68^{\circ} \mathrm{C}$, corresponding to melting point of COM followed by the flat profile and then the reduced endothermic peak of MET at $233^{\circ} \mathrm{C}$. This change in the melting points of both the components may be attributed due to the dilution factor. The thermal curve of SC4 [Figure 4d] displayed a broad endothermic peak ranging from $55^{\circ} \mathrm{C}$ to $59^{\circ} \mathrm{C}$ followed by endothermic and exothermic curves and finally the endothermic peak at $231^{\circ} \mathrm{C}$, due to MET.

\section{X-ray powder diffractometry}

Figure 5 shows the powder XRD patterns of the pure drug, Compritol 888 ATO, physical mixture (1:3), and solid dispersion prepared by solvent evaporation (SC4) with compritol 888 ATO. In powder X-ray diffraction, sharper diffraction peaks indicate more crystalline material. The sharp, intense representative peaks of pure MET [Figure 5a] notably at $2 \theta$ angles were $17^{\circ}, 22^{\circ}, 23^{\circ}, 31^{\circ}$, and $45^{\circ}$. This series of sharp and intense diffraction peaks indicated the crystalline state of pure MET. The polymorphic structure of a drug is an important parameter that influences the dissolution rate and bioavailability of the drug. Compritol shows two peaks [Figure 5b] that occur due to lipidic polymorphism at $21^{\circ}$ and $23^{\circ}$. The diffraction pattern of the physical mixtures [Figure 5c] was simply the superimposition of those of the pure components. In case of solid dispersion prepared by the solvent evaporation method [Figure 5d] the diffraction patterns were very similar to those of the physical mixture showing all the intense peaks of both the drug and polymer.

\section{Stability studies}

No visible changes in the appearance of the solid dispersion were observed at the end of the storage period. The drug content and dissolution of metformin was almost similar to that at time zero during the whole period of investigation.

\section{DISCUSSION}

It was observed in UV studies that there was slight reduction in the absorbance of MET in solid dispersion

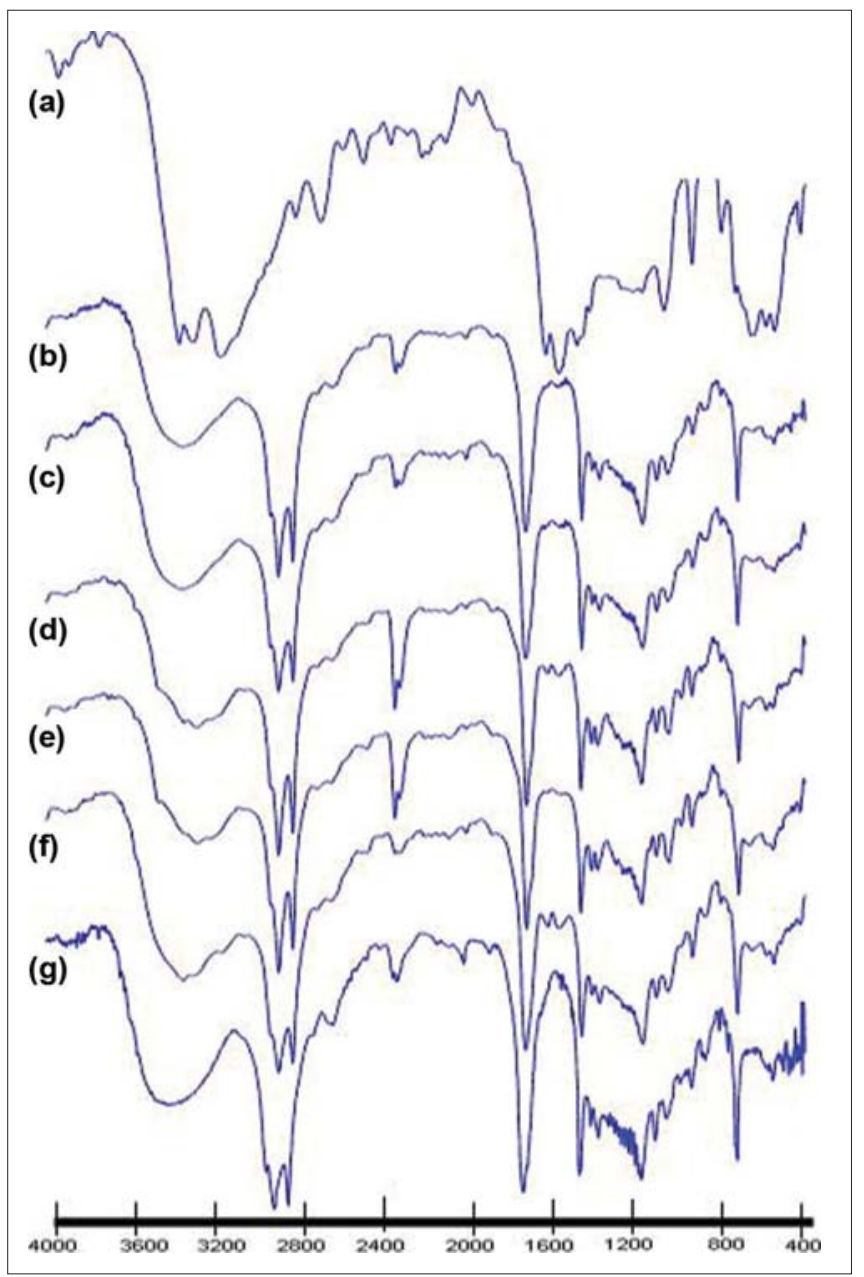

Figure 3: FTIR spectra of (a) MET, (b) CC5, (c) CC4, (d) SC5, (e) SC4, (f) $\mathrm{PC}$, and (g) compritol

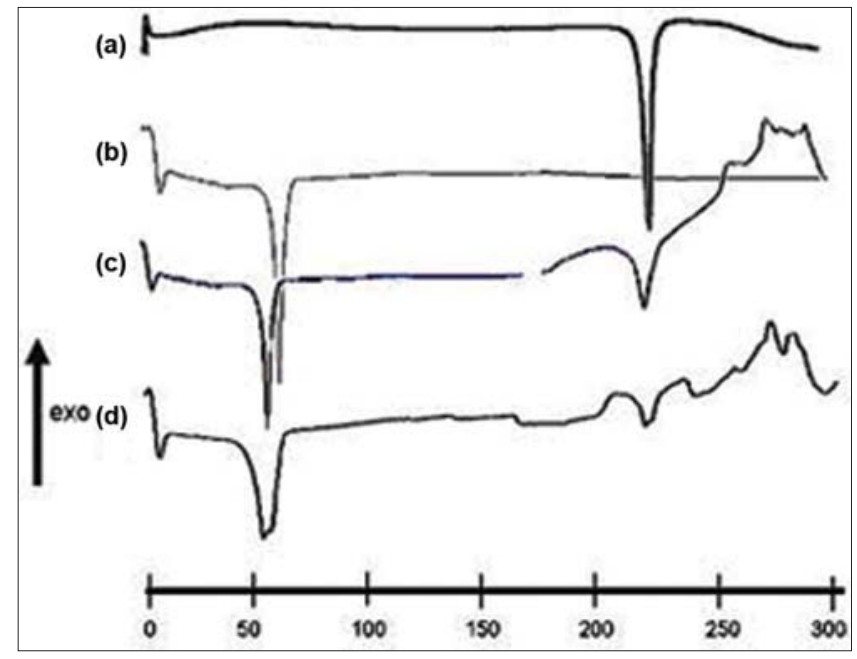

Figure 4: DSC curves of (a) metformin hydrochloride, (b) compritol, (c) physical mixture, (d) SC4 SD 


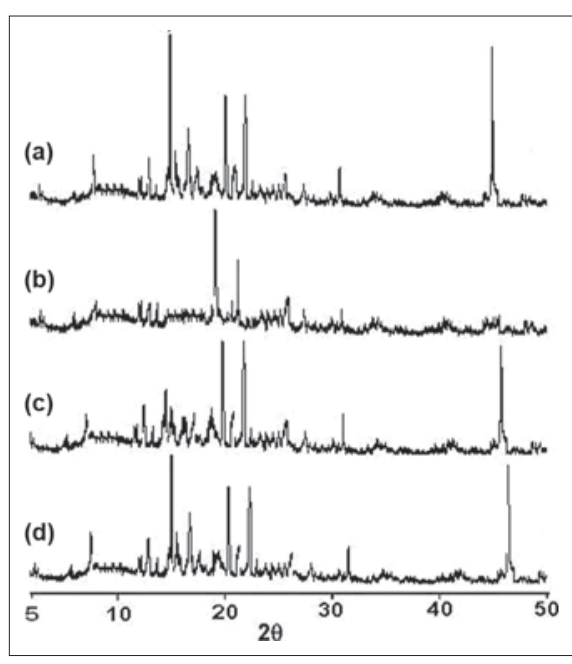

Figure 5: XRD patterns of (a) metformin hydrochloride, (b) compritol, (c) physical mixtures, (d) SC4 SD

than the pure drug. This induced change in absorbance was attributed, primarily, to the weak hydrogen bonding.

The in vitro dissolution study showed the slow drug release from SD, namely, SC3, SC4, and SC5, which was due to an increase in the overall lipophilicity of solid dispersions, leading to a decrease in the effective interfacial area between the drug and the dissolution medium. This resulted in the reduction of the dispersion wettability (the rate of the dissolution medium penetration into the solid dispersions) and consequently led to a decrease in drug diffusion from the dispersions. SC3 showed a sustained release effect with $92 \%$ drug release after 10 hours, but could fit into the release criteria as per USP test 2. SC4 solid dispersions followed the drug release pattern as per the required conditions and was considered as the idle ratio as per USP test 2. Further increase in the amount of the polymer resulted in the retarded release of the drug with just $82 \%$ drug release for SC5 after 10 hours, but the required release was not less than $85 \%$ after 10 hours, hence, ruling out the probability of fitting into the release criteria. Initial burst release (more than $60 \%$ in the first hour) from all the solid dispersions prepared by the closed melt method could probably be attributed to the dissolution of the drug from the surface of the dispersions, indicating failure of formation of dispersions. None of the solid dispersions showed promising results in sustaining the drug release for 10 hours.

The dissolution efficiency values are consistent with the dissolution data. For example, the DE value for the SD SC1 was $88.11 \%$, whereas, this value decreased to 63.90 and $60.60 \%$ for the SDs SC4 and SC5, respectively, for $\mathrm{SDs}$ prepared by the solvent evaporation method. The DE values were 91.13 and $86.28 \%$ for CC1 and CC5, respectively. The $\mathrm{DE}$ values do not show any major changes in case of solid dispersions with compritol using the closed melt method.

The kinetic studies when applied to solid dispersion showed that the release profile followed was the Korsmeyer-Peppas equation. The diffusion mechanism of drug release from SC4 was further confirmed by the Korsmeyer-Peppas plots that showed fair linearity $\left(\mathrm{R}^{2}>0.98\right)$, with a slope value of 0.3206 , which was far less than 0.5 , suggesting that the drug release mechanism from the selected solid dispersions was diffusion controlled. From the similarity factor data it could be concluded that there was no significant difference in the release profile of the prepared solid dispersion and the marketed tablets of MET.

In the IR studies there was no sign of any bands, which had disappeared, suggesting that the bonding, if present, may not have been so strong as to suppress the band. The crests and troughs associated with the SC4 SD indicated some kind of interaction between the drug and polymer. However, the presence of melting peaks in the curve of the solid dispersion indicated the absence of drug-carrier interactions and/or drug inclusion complexation. The DSC and XRD studies proved that there was retention of the crystalline nature of the drug in solid dispersion ruling out any probability of drug and polymer interaction or complex formation.

\section{CONCLUSION}

Solid dispersions for the controlled release of MET were developed by using compritol. The release rate of MET from SD was significantly affected by the preparation technique, used for obtaining solid dispersion, and also the quantity of polymer in the system. The solvent evaporation method was found to be more helpful than the closed melt in giving the required sustained release action. SD containing 80\% Compritol (SC4) showed suitable release kinetics, and was free of any interaction between the polymers and drug. Drug release from SC4 SD followed the Korsmeyer-Peppas equation. The FTIR and UV results did not show any drug-wax interaction. DSC and PXRD studies ruled out the occurrence of solid state interaction and complex formation. From the similarity factor data it could be concluded that there was no significant difference in the release profile of the solid dispersions and the marketed tablets of metformin hydrochloride. Therefore, SC4 $\mathrm{SD}$ was selected as the most effective candidate for the subsequent development of a well-timed, sustainedrelease dosage form of the drug. 


\section{REFERENCES}

1. Corti G, Capasso G, Maestrelli F, Cirri M, Mura P. Physical-chemical characterization of binary systems of metformin hydrochloride with triacetyl- $\beta$-cyclodextrin. J Pharm Biomed Anal 2007;45:480-6.

2. Mandal U, Gowda V, Ghosh A, Bose A, Bhaumik U, Chatterjee B, et al. Optimization of metformin hcl $500 \mathrm{mg}$ sustained release matrix tablets using artificial neural network based on multilayer perceptrons model. Chem Pharm Bull (Tokyo) 2008;56:150-5.

3. Lordi NG. Sustained release dosage forms. In : Lachman L, Lieberman HA, Kanig JL, editors. The theory and practice of industrial pharmacy. Bombay : Varghese Publishing House; 1990. p. 430-56.

4. Suzuki H, Sunada H. Influence of water-soluble polymers on the dissolution of nifedipine solid dispersions with combined carriers. Chem Pharm Bull (Tokyo) 1998;46:482-7.

5. Ozeki T, Yuasa H, Kanaya Y, Oishi K. Application of the solid dispersion method to controlled release of medicine. VIII. Medicine release and viscosity of the hydrogel of a water soluble polymer in a three-component solid dispersion system. Chem Pharm Bull 1995;43:1574-9.

6. Ozeki T, Yuasa H, Okada H. Controlled release of drug via methylcellulosecarboxyvinylpolymer interpolymer complex solid dispersion. AAPS Pharm Sci Tech 2005;6:E231-6.

7. Shivkumar HN, Desai BG, Deshmukh G. Design and optimization of diclofenac sodium controlled release solid dispersions by response surface methodology. Indian J Pharm Sci 2008;70:22-30.

8. Reza SM, Quadir MA, Haider SS. Comparative evaluation of plastic, hydrophobic and hydrophilic polymers as matrices for controlled-release drug delivery. J Pharm Pharm Sci 2003;6:282-91.

9. Tiwari SB, Murthy TK, Pai MR, Mehta PR, Chowdary PB. Controlled release formulation of tramadol hydrochloride using hydrophilic and hydrophobic matrix system. AAPS Pharm Sci Tech 2003;4:1-6:E31.

10. Barthelemy P, Laforet JP, Farah N, Joachim J. Compritol 888 ATO : An innovative hot-melt coating agent for prolonged release drug formulations. Eur J Pharm Biopharm 1999;47:87-90.

11. Faham A, Prinderre P, Piccerelle P, Farah N, Joachim J. Hot melt coating technology : Influence of compritol 888 ATO and granule size on chloroquine release. Pharmazie 2000;55:444-8.

12. Hamdani J, Moes AJ, Amighi K. Physical and thermal characterisation of precirol and compritol as lipophilic glycerides used for the preparation of controlled-release matrix pellets. Int J Pharm 2003;260:47-57.

13. Zhang YE, Schwartz JB. Melt granulation and heat treatment for wax matrix-controlled drug release. Drug Dev Ind Pharm 2003;29:131-8.

14. Brubach JB, Jannin V, Mahler B, Bourgaux C, Lessieur P, Roy P, et al. Structural and thermal characterization of glyceryl behenate by $\mathrm{x}$-ray diffraction coupled to differential calorimetry and infrared spectroscopy. Int J Pharm 2007;336:248-56.

15. Dabbagh MA, Taghipour B. Investigation of solid dispersion technique in improvement of physicochemical characteristics of ibuprofen powder. Iran J Pharm Sci 2007;3:69-76.
16. Xie Y, Xie P, Song X, Tang X, Song H. Preparation of esomeprazole zinc solid dispersion and study on its pharmacokinetics. Int $\mathrm{J}$ Pharm 2008;360:53-7.

17. Furuyama N, Hasegawa S, Hamaura T, Yada S, Nakagami H, Yonemochi E, et al. Evaluation of solid dispersions on a molecular level by the Raman mapping technique. Int J Pharm 2008;361:12-8.

18. Chauhan B, Shimpi S, Paradkar A. Preparation and characterization of etoricoxib solid dispersions using lipid carriers by spray drying technique. AAPS PharmSciTech 2005;6:E405-12.

19. Chowdary KP, Nalluri BN. Studies on nimesulide and $\beta$ cyclodextrin inclusion complexes. Indian Drugs 2000;37:299-304.

20. Stepensky D, Friedman M, Srour W, Raz I, Hoffman A. Preclinical evaluation of pharmacokinetic-pharmacodynamic rationale for oral CR metformin formulation. J Control Release 2001;71:107-15.

21. US Pharmacopoeia XXXII. First supplement. Metformin hydrochloride extended release tablets Monograph. Rockville, MD : US Pharmacopeial Convention; 2009. p. 4073-7.

22. Nokhodchi A, Hassan-Zadeh D, Monajjem-Zadeh F, Taghi-Zadeh N. Effect of various surfactants and their concentration on controlled release of captopril from polymeric matrices. Acta Pharm 2008;58:151-62.

23. Ali J, Arora S, Ahuja A, Babbar AK, Sharma RK, Khar RK, et al. Formulation and development of hydrodynamically balanced system for metformin: In vitro and in vivo evaluation. Eur J Pharm Biopharm 2007;67:196-201.

24. Korsmeyer RW, Gurny R, Doelker E, Buri P, Peppas NA. Mechanisms of solute release from porous hydrophilic polymers. Int J Pharm 1983;15: 25-35.

25. Avachat A, Kotwal V. Design and evaluation of matrix-based controlled release tablets of diclofenac sodium and chondroitin sulphate. AAPS Pharm Sci Tech 2007;8:E1-E88.

26. Bolton S, Bon C. Quality control. Pharmaceutical statistics : Practical and clinical applications. $4^{\text {th }}$ ed. Vol 135. New York : Marcel Dekker; 2004. p. $408-11$.

27. Nokhodchi A, Talari R, Valizadeh H, Jalali MB. An investigation on the solid dispersions of chlordiazepoxide. Int J Biomed Sci 2007;3:211-7.

28. Samuel B, Naim S, Chauhan B, Mahadik KR, Paradkar A. Preparation and characterization of metformin hydrochloride - $\lambda$ carrageenan complex. Indian Drugs 2004;41:655-60.

29. Farago PV, Raffin RP, Pohlmann AR, Guterres SS, Zawadzki SF. Physicochemical characterization of a hydrophilic model drug-loaded PHBV Microparticles obtained by the double emulsion/solvent evaporation technique. J Braz Chem Soc 2008;19:1298-305.

30. Mathews BR. Regulatory aspects of stability testing in Europe. Drug Dev Ind Pharm 1999;25:831-56.

How to cite this article: Jagdale SC, Patil SA, Kuchekar BS, Chabukswar AR. Preparation and characterization of metformin hydrochloride - Compritol 888 ATO solid dispersion. J Young Pharmacists 2011;3:197-204.

Source of Support: Nil, Conflict of Interest: None declared.

\section{Announcement}

\section{"Quick Response Code" link for full text articles}

The journal issue has a unique new feature for reaching to the journal's website without typing a single letter. Each article on its first page has a "Quick Response Code". Using any mobile or other hand-held device with camera and GPRS/other internet source, one can reach to the full text of that particular article on the journal's website. Start a QR-code reading software (see list of free applications from http://tinyurl.com/ yzlh2tc) and point the camera to the QR-code printed in the journal. It will automatically take you to the HTML full text of that article. One can also use a desktop or laptop with web camera for similar functionality. See http://tinyurl.com/2bw7fn3 or http://tinyurl.com/3ysr3me for the free applications. 